\title{
Prospective exploratory study of gemcitabine and $S-1$ against elderly patients with advanced non-small cell lung cancer
}

\author{
KYOICHI KAIRA $^{1,2}$, NORIKO YANAGITANI ${ }^{3}$, NORIAKI SUNAGA ${ }^{2,4}$, HISAO IMAI $^{5}$, AKIHIRO ONO $^{2}$, \\ YASUHIKO KOGA $^{2}$, TAKESHI HISADA ${ }^{2}$, TAMOTSU ISHIZUKA ${ }^{6}$ and MASANOBU YAMADA ${ }^{2}$ \\ Departments of ${ }^{1}$ Oncology Clinical Development and ${ }^{2}$ Medicine and Molecular Science, \\ Gunma University Graduate School of Medicine, Gunma University Hospital, Maebashi, Gunma 371-8511; \\ ${ }^{3}$ Department of Thoracic Medical Oncology, Cancer Institute Hospital, Japanese Foundation for Cancer Research, \\ Tokyo 135-8550; ${ }^{4}$ Oncology Center, Gunma University Hospital, Maebashi, Gunma 371-8511; \\ ${ }^{5}$ Department of Respiratory Medicine, Gunma Prefectural Cancer Center, Ota, Gunma 373-8550; \\ ${ }^{6}$ Third Department of Internal Medicine, University of Fukui, Yoshida, Fukui 910-1193, Japan
}

Received October 4, 2016; Accepted March 3, 2017

DOI: $10.3892 / 01.2017 .6259$

\begin{abstract}
A prospective study was conducted to investigate the efficacy of a combined regimen of gemcitabine and S-1 for the treatment of elderly patients ( $>70$ years) with advanced non-small cell lung cancer (NSCLC) as a first-line setting based on the dosage recommended in a previous phase I study. Chemotherapy-naïve patients with advanced NSCLC received gemcitabine plus $\mathrm{S}-1 . \mathrm{S}-1\left(40 \mathrm{mg} / \mathrm{m}^{2}\right)$ was administered orally twice daily for 14 days while gemcitabine $\left(1,000 \mathrm{mg} / \mathrm{m}^{2}\right)$ was administered on days 1 and 15 of each cycle, and this regimen was repeated every 4 weeks. A total of 20 patients were included in the present study. Of these, 8 patients achieved an overall response rate of $40.0 \%$, and the overall disease control rate was $65.0 \%$. According to the histological type, the response rate in patients with NSCLC and adenocarcinoma was $38.5 \%$, and that for non-adenocarcinoma was $42.9 \%$. Progression-free survival and median survival times were 6.4 months and 17.8 months, respectively. Grade 3 or 4 hematological toxicities observed were leukopenia (29\%) and neutropenia (24\%), while febrile neutropenia was not observed in any patient. The only non-hematological adverse event observed was grade 3 skin rash $(10 \%)$. Therefore, the combination of gemcitabine and S-1 may be a promising and feasible regimen in the first-line setting for elderly patients with advanced NSCLC.
\end{abstract}

Correspondence to: Dr Kyoichi Kaira, Department of Oncology Clinical Development, Gunma University Graduate School of Medicine, Gunma University Hospital, 3-39-22 Showa-machi, Maebashi, Gunma 371-8511, Japan

E-mail: kkaira1970@yahoo.co.jp

Key words: non-small cell lung cancer, elderly patient, gemcitabine, $\mathrm{S}-1$, efficacy, prospective study

\section{Introduction}

Lung cancer is the leading cause of cancer-associated mortality worldwide. Lung cancer is classified into non-small cell lung cancer (NSCLC) and small-cell lung cancer, and $80 \%$ of lung cancers are NSCLC (1). Patients with early stage NSCLC are candidates for curative thoracic surgery, whereas those with advanced stage NSCLC are usually treated with systemic chemotherapy or chemoradiotherapy. In total, $50 \%$ of patients with NSCLC are diagnosed with stage IIIB or IV cancers, and morbidity is higher among elderly patients compared with non-elderly patients (1). Previous meta-analyses have revealed that platinum-based combination chemotherapy slightly improves survival compared with best supportive care $(2,3)$. A previous study compared the survival time of elderly patients with NSCLC who received single-agent treatment, with that of patients who underwent a platinum-based regimen, and median overall survival was 10.3 months for platinum-based chemotherapy and 6.2 months for monotherapy $(\mathrm{P}<0.0001)$. The 1-year survival rate was $44.5 \%$ and $25.4 \%$, respectively (4). The incidence of lung cancer has been increasing among elderly individuals, and single agents including vinorelbine and docetaxel have been widely used as first-line treatments against NSCLC in elderly patients with advanced disease in Japan. In a phase III study, Abe et al (5) reported that the survival time following docetaxel monotherapy was significantly increased compared with combination therapy with docetaxel plus cisplatin in elderly patients with advanced NSCLC. Therefore, it remains unclear whether combination chemotherapy improves survival compared with monotherapy in these patients. Further studies evaluating the clinical significance of combination chemotherapy against advanced NSCLC in elderly patients are warranted.

S-1 (Taiho Pharmaceutical Co., Ltd., Tokyo, Japan) is an oral anticancer agent composed of tegafur, 5-chloro-2, 4-dihydroxypyridine, and potassium oxonate, in a molar ratio of 1:0.4:1 (6). Tegafur, a prodrug of 5-fluorouracil (5-FU), is gradually converted to 5-FU, and is rapidly catabolized 
by dihydropyrimidine dehydrogenase in the liver. In several studies, S-1 has been reported to actively reduce tumor growth in various human cancers, and a combination of platinum-based regimens with $\mathrm{S}-1$ has been the standard treatment regimen against advanced NSCLC (7-12). Gemcitabine, an anticancer drug that structurally resembles cytosine arabinoside, has been demonstrated to exhibit high anti-tumor activity with minimal adverse effects (13). A previous study revealed that a combination of gemcitabine and uracil-tegafur was effective and tolerable in patients with advanced NSCLC (14). It was demonstrated that a combination of gemcitabine and S-1 was also useful in patients with advanced pancreatic cancer (15). Therefore, S-1 and gemcitabine are considered to be antimetabolites with minimal toxicities and promising efficacies.

In a previous phase I study, a combination of gemcitabine and S-1 was observed to be an effective and feasible treatment against NSCLC in elderly patients (16). Seto et al (17) also reported the clinical benefit of gemcitabine plus S-1 in elderly patients, with the combination regimen yielding a response rate of $27 \%$, a time to progression of 4.2 months and an overall survival of 12.9 months, with minimum toxicity. Their treatment schedule was the oral administration of S-1 (30 mg/m² twice a day) on days $1-14$, and intravenous administration of gemcitabine $\left(1,000 \mathrm{mg} / \mathrm{m}^{2}\right)$ on days 8 and 15. On the other hand, the treatment schedule followed in the previous study performed by our group was the oral administration of S-1 (40 mg/m² twice a day) on days $1-14$, and intravenous administration of gemcitabine $\left(1,000 \mathrm{mg} / \mathrm{m}^{2}\right)$ on days 1 and 15. The dose of S-1 and the administrative schedule of gemcitabine differed between the previous study by our group and the study by Seto et al (17). In another study, Satouchi et al (18) recommended two treatment schedules that differed in the administration schedule of gemcitabine: The oral administration of $\mathrm{S}-1\left(30 \mathrm{mg} / \mathrm{m}^{2}\right.$ twice a day) on days 1-14 and intravenous administration of gemcitabine $\left(1,000 \mathrm{mg} / \mathrm{m}^{2}\right)$ on days 1 and 8 or days 8 and 15 in patients with chemotherapy-naïve NSCLC. Similarly, Takiguchi et al (19) also described the oral administration of $\mathrm{S}-1\left(30 \mathrm{mg} / \mathrm{m}^{2}\right.$ twice a day) on days 1-14 and intravenous administration of gemcitabine $\left(1,000 \mathrm{mg} / \mathrm{m}^{2}\right)$ on days 8 and 15 . However, it remains unclear whether the differences in S-1 dose and gemcitabine administration affected the response rate and survival time in patients with advanced NSCLC. Therefore, the present study was conducted to investigate the efficacy of the regimen comprised of S-1 plus gemcitabine based on the dosage used in the previous phase I study performed by our group (16).

\section{Patients and methods}

Patients and patient eligibility. A total of 21 patients were enrolled in the present study between August 2007 and March 2015 at Gunma University Hospital (Maebashi, Japan) A single patient withdrew because of the occurrence of vascular disease. Patient characteristics are detailed in Table I. The inclusion criteria were histologically and/or cytologically proven unresectable stage IIIB or IV NSCLC $(1,3)$, no previous systemic chemotherapy or radiotherapy, an Eastern Cooperative Oncology Group (ECOG) performance status (PS) of 0 or 1 , age $\geq 70$ years, a life expectancy of $\geq 12$ weeks; adequate bone marrow reserve (leukocyte count $\geq 4,000 \mathrm{~mm}^{-3}$, neutrophil count $\geq 2,000 \mathrm{~mm}^{-3}$, platelet count $\geq 100,000 \mathrm{~mm}^{-3}$ and hemoglobin $\geq 10 \mathrm{~g} / \mathrm{dl}$ ), normal liver function (total serum bilirubin $\leq 1.5 \mathrm{mg} / \mathrm{dl}$, and aspartate transaminase, alanine transaminase $<2 x$ the upper limits of the normal range), regardless of epidermal growth factor receptor $(E G F R)$ mutation status and normal renal function (serum creatinine $\leq 1.5 \mathrm{mg} / \mathrm{dl}$ and creatinine clearance $\geq 60 \mathrm{ml} / \mathrm{min}$ ). Patients with concomitant malignancies, central nervous system metastases, active infectious diseases or other serious medical problems were excluded. The institutional Review Board at Gunma University Hospital approved the present study, and written informed consent was obtained from all patients (approval no. UMIN000001750).

Clinical study design. The present study was a prospective, single-center, single-arm study investigating the effectiveness of gemcitabine and S-1 combination therapy for the treatment of elderly patients with NSCLC. S-1 $\left(80 \mathrm{mg} / \mathrm{m}^{2} /\right.$ day $)$ was administered orally twice daily following a meal for 14 consecutive days, followed by 2 weeks without treatment. Each S-1 capsule contained 20 or $25 \mathrm{mg}$ tegafur. Individual doses were rounded down to the nearest pill size less than the calculated dose, given the available formulation. Gemcitabine $\left(1,000 \mathrm{mg} / \mathrm{m}^{2} /\right.$ day $)$ was administered as a 30 -min intravenous infusion on days 1 and 15 of each cycle. The cycle was repeated every 4 weeks. Although the prophylactic administration of granulocyte-colony stimulating factor (G-CSF) was not permitted, the administration of G-CSF was permitted in patients with grade 4 neutropenia and/or grade 3 febrile neutropenia. Subsequent cycles of chemotherapy were initiated when the leukocyte counts were $\geq 4,000 \mathrm{~m}^{-3}$, and the platelet counts were $\geq 100,000 \mathrm{~m}^{-3}$ following day 29 . If the leukocyte or platelet counts had not returned to these levels by day 1 of the next cycle of chemotherapy, the drugs were withheld until full recovery. Chemotherapeutic treatment was performed for at least two cycles, unless unacceptable toxicity or disease progression occurred.

Treatment assessment. Patients were evaluated prior to treatment with complete blood cell count evaluation, differential count evaluation, routine chemistry measurements, chest radiography, chest computed tomography (CT), abdominal CT, whole-brain magnetic resonance imaging or CT, and isotope bone scintigraphy. Evaluations performed weekly were complete blood cell count, differential count, routine chemistry measurements, physical examination, and toxicity assessment. Response Evaluation Criteria in Solid Tumors version 1.1 was used to assess the response to S-1 plus gemcitabine (20). To evaluate the response, CT scans were performed every 6 weeks until progressive disease developed. The overall response was defined as the best response. Second-line chemotherapy or other treatments following the present study were not prohibited by the protocol. Adverse events were assessed according to the Common Terminology Criteria for Adverse Events version 3.0 (21).

Statistical analysis. The primary endpoint of the present study was to evaluate the overall response rate (ORR), and the secondary endpoints were to examine the adverse events and 
survival data. Progression-free survival (PFS) was defined as the time from treatment initiation to disease progression or mortality. Overall survival (OS) was determined as the time from the start of the treatment to mortality from any cause. Survival estimation was performed using the Kaplan-Meier method and the log-rank test. $\mathrm{P}<0.05$ was considered to indicate a statistically significant difference. The estimated required accrual was 19 patients, assuming an ORR of $35 \%$ in eligible patients would indicate potential usefulness, whereas an ORR of $13 \%$ would constitute the lower limit of interest, with a power of $80 \%$ and $\alpha$ level set at $5 \%$ (one-sided test). The estimates were based on the $32.7-47.0 \%$ response rates reported in previous trials for platinum-based combination regimens with S-1 $(6,7)$ and $12.5-22.7 \%$ response rates reported by a study conducted using S-1 monotherapy (8.9). All statistical analyses were performed using GraphPad Prism 4 software (GraphPad Software, La Jolla, CA, USA) for Microsoft Windows.

\section{Results}

Patient demographics. Patient characteristics are detailed in Table I. The median age of patients was 78 years (range, 70-86 years); $10(50 \%)$ patients were men, and $10(50 \%)$ were women. Histology indicated $13(65 \%)$ adenocarcinomas, $5(25 \%)$ squamous cell carcinoma and $2(10 \%)$ other. Two patients (10\%) had stage IIIB disease, and 18 (90\%) had stage IV disease. Other demographics included an ECOG performance status score of $0(80 \%)$ and a history of smoking (60\%). The distribution of comorbid diseases was as follows: 4 patients with chronic obstructive pulmonary disease; 5 patients with hypertension (medically treated); 4 patients with diabetes mellitus; 2 patients with arrhythmia; and 2 patients with angina pectoris (medically treated). The status of EGFR mutation was assessed in 13 patients with adenocarcinoma. Of these 13 patients, there were 4 patients with EGFR mutation, 7 patients with EGFR wild type and the other 2 patients had unknown status.

Treatment delivery. Chemotherapy was administered to 20 patients, and the median number of cycles was 2 (range, $1-27$ ). Four or more cycles were administered to $\sim 35 \%$ of all patients. Among the total 74 cycles administered, gemcitabine was not skipped in any of the patients. S-1 was administered at $>98 \%$ of the scheduled dosage in all cycles. Following disease progression, 9 patients received second- or third-line chemotherapy including gefitinib or erlotinib. The 4 patients harboring EGFR mutations were treated with gefitinib or erlotinib, and the 5 patients with $E G F R$ wild type received erlotinib.

Efficacy and survival data. A total of 17 patients completed $>2$ cycles of chemotherapy. Three patients discontinued treatment prematurely following 1 cycle due to adverse effects and patient choice. None of the patients achieved complete response (CR), and 8 achieved a partial response (PR) with an ORR of $40.0 \%$ [95\% confidence interval (CI): 18.5-61.5\%]. The overall disease control rate $(\mathrm{CR}+\mathrm{PR}+$ stable disease $)$ was $65.0 \%$ (95\% CI: 44.1-85.9\%; Table II). According to the histological type, patients with adenocarcinoma exhibited a response rate of $38.5 \%$ (95\% CI: $12.0-64.9 \%$; Table II) and
Table I. Patient demographics

\begin{tabular}{lc}
\hline Clinical characteristic & Value \\
\hline Age [years; median (range)] & $78(70-86)$ \\
Sex (male/female) & $10 / 10$ \\
ECOG PS (0/1) & $16 / 4$ \\
Histology (AC/SQC/other) & $13 / 5 / 2$ \\
Clinical stage (IIIB/IV) & $2 / 18$ \\
Smoking history (yes/no) & $12 / 8$ \\
Comorbid disease (yes/no) & $17 / 3$ \\
Recurrence following operation (yes/no) & $5 / 15$ \\
\hline
\end{tabular}

ECOG, European clinical oncology group; PS, performance status; $\mathrm{AC}$, adenocarcinoma; SQC, squamous cell carcinoma.

Table II. Response rate according to histological type.

\begin{tabular}{lccccc}
\hline & & \multicolumn{4}{c}{ Response } \\
\cline { 3 - 6 } Histology & $\begin{array}{c}\text { No. } \\
\text { of patients }\end{array}$ & CR & PR & SD & PD \\
\hline AC & 13 & 0 & 5 & 4 & 4 \\
Non-AC & 7 & 0 & 3 & 1 & 3 \\
Total patients & 20 & 0 & 8 & 5 & 7 \\
Response rate of & & & $40.0 \%$ & \\
total patients & & $(95 \%$ CI; 18.5-61.5\%) \\
$\begin{array}{l}\text { Disease control rate } \\
\text { of total patients }\end{array}$ & & \multicolumn{4}{c}{$65.0 \%$} \\
\hline $\begin{array}{l}\text { CR, complete response; PR, partial response; SD, stable disease; PD, } \\
\text { progressive disease; CI, confidence interval; AC, adenocarcinoma. }\end{array}$ \\
\hline
\end{tabular}

those without adenocarcinoma exhibited a response rate of 42.9\% (95\% CI: 6.2-79.5\%; Table II). The median PFS was 6.4 months (95\% CI: 4.0-17.0), and the PFS rates at 3 and 6 months were 85.0 and $48.2 \%$, respectively (Fig. 1A). The median survival time (MST) was 17.8 months $(95 \% \mathrm{CI}$ : 6.0-46.0), and the OS rates at 6 and 12 months were 78.8 and $59.3 \%$, respectively (Fig. 1B).

Toxicity. Adverse events were assessed in all the treated patients. Hematological and non-hematological adverse events are listed in Table III. Grade 3 or 4 hematological toxicities (21) were leukopenia $(30 \%)$, neutropenia $(25 \%)$, anemia $(0 \%)$, and thrombocytopenia ( $0 \%$ ). Febrile neutropenia was not observed in any patients. The only non-hematological adverse event observed was grade 3 skin rash (10\%). Pulmonary injuries, including interstitial pneumonia, and treatment-associated mortality were not observed in the present study.

\section{Discussion}

In the present study, a combination of gemcitabine and S-1 was demonstrated to be feasible and effective as a first-line 
Table III. Hematological and non-hematological adverse events.

\begin{tabular}{lccccc}
\hline & \multicolumn{5}{c}{ Grade } \\
\cline { 2 - 6 } Adverse event & 1 & 2 & 3 & 4 & 3 or $4(\%)$ \\
\hline Leukopenia & 2 & 3 & 5 & 1 & 29 \\
Neutropenia & 2 & 2 & 4 & 1 & 24 \\
Febrile neutropenia & 0 & 0 & 0 & 0 & 0 \\
Anemia & 5 & 4 & 0 & 0 & 0 \\
Thrombocytopenia & 4 & 2 & 0 & 0 & 0 \\
Nausea/vomiting & 2 & 1 & 0 & 0 & 0 \\
Anorexia & 5 & 1 & 0 & 0 & 0 \\
Diarrhea & 1 & 0 & 0 & 0 & 0 \\
Liver dysfunction & 1 & 0 & 0 & 0 & 0 \\
Infection & 1 & 0 & 0 & 0 & 0 \\
Skin rash & 5 & 1 & 2 & 0 & 10 \\
Constipation & 0 & 1 & 0 & 0 & 0 \\
Fever & 5 & 0 & 0 & 0 & 0 \\
Neuropathy-sensory & 0 & 0 & 0 & 0 & 0 \\
Pneumonitis & 3 & 2 & 0 & 0 & 0 \\
Vertigo & 0 & 0 & 0 & 0 & 0 \\
Alopecia & 0 & 0 & 0 & 0 & 0 \\
Fatigue & 3 & 1 & 0 & 0 & 0 \\
\hline
\end{tabular}
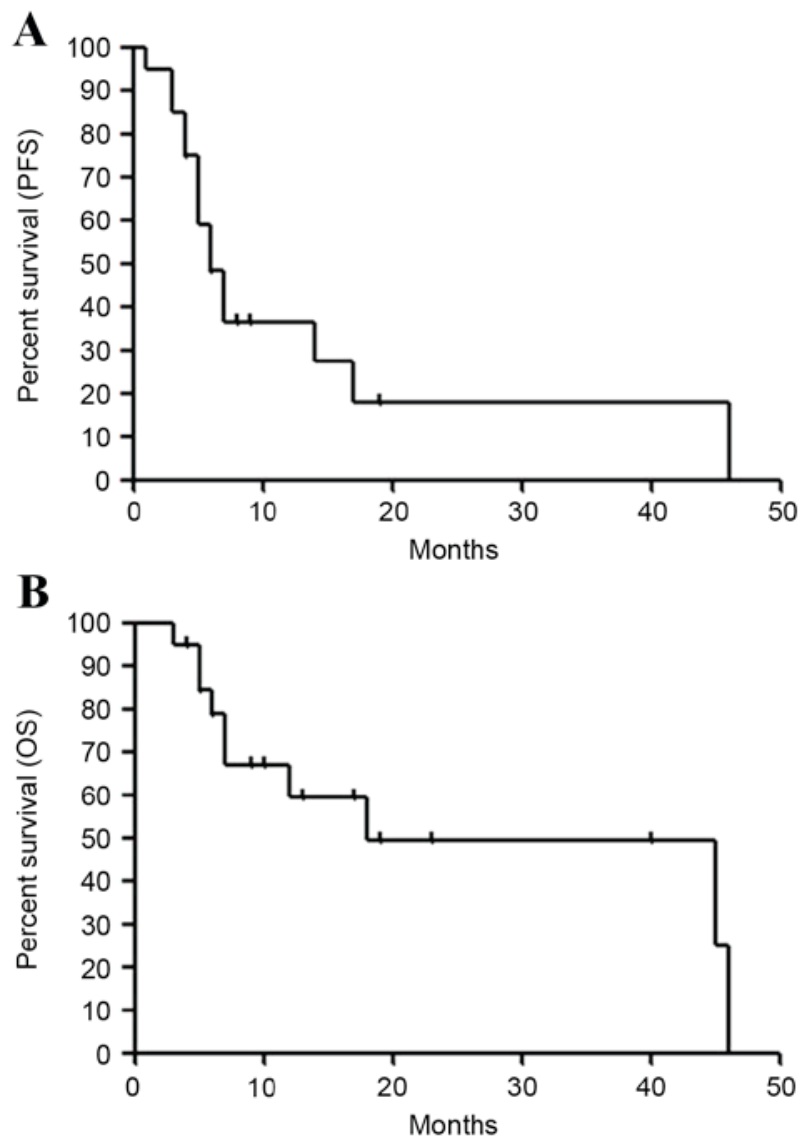

Figure 1. Kaplan-Meier survival curves for (A) PFS and (B) OS for all patients. Median PFS was 6.4 months, and median survival time was 17.8 months. PFS, progression free survival; OS, overall survival. treatment in elderly patients with advanced NSCLC. A previous phase I study of this regimen reported mild toxicities and a response rate of $42.9 \%$ (16). The response rate in the present study almost coincided with that of this previous phase I study. To date, only three studies have reported the combination of gemcitabine and S-1 in the treatment of patients with advanced NSCLC (Table IV) (17-19). In a previous prospective study conducted in a first-line setting, two regimens were investigated, and the efficacy was 22.0 and $28.9 \%$, the PFS was 3.6 and 4.1 months, and the OS was 15.5 and 18.8 months, with mild toxicities (18). A study reporting a platinum-refractory case yielded a response rate of $23.5 \%$, a PFS of 6.6 months and an OS of 19.9 months (19). A phase II trial in elderly patients with NSCLC indicated a response rate of $27 \%$, a time to progression of 4.2 months, and an OS of 12.9 months (17). The present study suggested a higher response rate associated with the regimen designed by our group compared with the other studies (17-19), although the sample size of the present study was very small. Regarding adverse events, the previous three studies demonstrated hematological toxicities in $45.9-61.0 \%$ with grade $3 / 4$ neutropenia, $21.0-28.9 \%$ with grade $3 / 4$ leukopenia and $4.9-13.5 \%$ with thrombocytopenia, and non-hematological toxicities in $0-6.0 \%$ with grade $3 / 4$ skin rash (17-19). Furthermore, febrile neutropenia was observed in $3.0-7.3 \%$ of patients, and grade $3 / 4$ pneumonia was observed in $4.9-9.0 \%$. On the other hand, the toxicities observed in the present study appeared to be mild compared with those observed in other studies with gemcitabine and S-1. Bi-weekly administration of gemcitabine plus S-1 may contribute to increased tolerability and efficacy, although the mechanisms underlying the effects of the regimen remain to be delineated. In addition, the survival data in the present study, although biased due to the small sample size, suggested that it was possible to compare the effectiveness of this regimen with that of the previous studies (17-19). Therefore, further studies conducted with a larger sample size to confirm the findings of the present study are warranted.

Single agents, including docetaxel or vinorelbine, have been recommended for the treatment of elderly patients with advanced NSCLC. In Japan, the results of phase III trials reported by Kudoh et al (22) and Abe et al (5) indicated that docetaxel was suitable for such patients. Kudoh et al (22) reported the OS, PFS and ORR of docetaxel were 14.3 months, 5.5 months and $22.7 \%$, respectively. In the phase III trial conducted by Abe et al (5) the ORR, MST and PFS were reported to be $24.6 \%, 14.3$ months and 4.4 months, respectively, which was superior to the results of survival data from platinum combination therapies including docetaxel. These studies had similar profiles of adverse events and grade 3/4 leukopenia was observed in $58.0-62.7 \%$, grade $3 / 4$ neutropenia in $82.9-88.8 \%$ and febrile neutropenia in $12.5-15.2 \%$ of the patients. The toxicities following docetaxel administration were markedly severe compared with those following gemcitabine plus S-1. However, the efficacy did not differ between docetaxel alone and gemcitabine plus S-1. In the present study, the ORR of gemcitabine plus S-1 may be superior to that of docetaxel alone irrespective of mild toxicities, comparable with that of the combination of platinum doublet. As anticancer 
therapies would be preferable in the outpatient rather than the inpatient treatment setting, the regimen examined in the present study may be appropriate for the treatment of advanced NSCLC, in particular in elderly patients with short life expectancies. Therefore, in terms of efficacy and tolerability, the administration of this regimen may be more effectively compared with previous treatment settings involving gemcitabine plus S-1 (17-19).

There are several limitations to the present study. Firstly, the selection procedure of patients eligible to be included in the present study took a long time (from 2007 to 2015 for only 20 patients). Therefore, a number of novel strategies have become available for the treatment of patients with advanced NSCLC, which may have rendered the survival time in the present study biased. Secondly, the treatment of all the patients was not judged according to the EGFR mutation status. It may be difficult for the results of the present study to indicate suitability for daily practice. A total of 4 patients harboring $E G F R$ mutations received gefitinib and erlotinib, thus, the efficacy of EGFR-tyrosine kinase inhibitors may affect the survival results. It is necessary to perform further studies on patients without EGFR mutations. Thirdly, the relationship between the efficacy of gemcitabine plus S-1 and any biomarkers, including thymidylate synthase or ribonucleotide reductase catalytic subunit M1, was not investigated. The discovery of any predictive biomarkers would improve the outcome following therapy. It is important to determine whether the efficacy of the regimen used in the present study would improve depending on the expression of any predictive markers. Finally, the sample size is limited and this may bias the results of the present study. Furthermore, the potential for effective treatment of elderly patients depends on physical function, mobility, nutrition, social support and the condition of comorbid diseases, thus, evaluating the individual patient is comprehensive and possible to achieve with a geriatric assessment in cooperation with PS (23). A previous report focused on the prognostic significance of a baseline assessment of functional status, comorbidity and quality of life and demonstrated that improved baseline quality of life and greater facility with activities of daily living were associated with a favorable outcome (24). In the present study, therefore, the absence of geriatric assessment may disturb the appropriate evaluation of the therapeutic efficacy against elderly patients. Further investigation and further studies using geriatric assessment are therefore warranted.

In conclusion, the results of the present study demonstrated that the combination of gemcitabine and S-1 was an effective and well-tolerated regimen in elderly patients with chemo-naïve advanced NSCLC. The treatment schedule followed in the present study seemed to be more effective compared with regimens evaluated in previous studies. Future studies comparing gemcitabine plus S-1 combination therapy to single-agent regimens including docetaxel or vinorelbine are warranted.

\section{Acknowledgements}

The authors would like to thank Ms. Yuka Matsui for her technical assistance during manuscript submission and Ms. Tomoko Okada for data collection and technical assistance. 


\section{References}

1. Siegel R, DeSantis C, Virgo K, Stein K, Mariotto A, Smith T, Cooper D, Gansler T, Lerro C, Fedewa S, et al: Cancer treatment and survivorship statistics, 2012. CA Cancer J Clin 62: 220-241, 2012.

2. Chemotherapy in non-small cell lung cancer: A meta-analysis using updated data on individual patients from 52 randomised clinical trials. Non-small Cell Lung Cancer Collaborative Group. BMJ 311: 899-909, 1995.

3. Azzoli CG, Temin S and Giaccone G: 2011 Focused Update of 2009 American Society of Clinical Oncology Clinical Practice Guideline Update on Chemotherapy for Stage IV Non-Small-Cell. Lung Cancer. J Oncol Pract 8: 63-66, 2012.

4. Quoix E, Zalcman G, Oster JP, Westeel V, Pichon E, Lavolé A, Dauba J, Debieuvre D, Souquet PJ, Bigay-Game L, et al: Carboplatin and weekly paclitaxel doublet chemotherapy compared with monotherapy in elderly patients with advanced non-small-cell lung cancer: IFCT-0501 randomized, phase 3 trial. Lancet 378: 1079-1088, 2011.

5. Abe T, Takeda K, Ohe Y, Kudoh S, Ichinose Y, Okamoto H, Yamamoto N, Yoshioka H, Minato K, Sawa T, et al: Randomized phase III trial comparing weekly docetaxel plus cisplatin versus docetaxel monotherapy every 3 weeks in elderly patients with advanced non-small-cell lung cancer: The intergroup trial JCOG0803/WJOG4307L. J Clin Oncol 33: 575-581, 2015.

6. Shirasaka T, Shimamato Y, Ohshimo H, Yamaguchi M, Kato T, Yonekura K and Fukushima M: Development of a novel form of an oral 5-fluorouracil derivative (S-1) directed to the potentiation of the tumor selective cytotoxicity of 5-fluorouracil by two biochemical modulators. Anticancer Drugs 7: 548-557, 1996.

7. Kubota K, Sakai H, Yamamoto N, Kunitoh H, Nakagawa K, Takeda K, Ichinose Y, Saijo N, Ariyoshi Y and Fukuoka M: A multi-institution phase I/II trial of triweeky regimen with S-1 plus cisplatin in patients with advanced non-small cell lung cancer. J Thorac Oncol 5: 702-706, 2010.

8. Ichinose Y, Yoshimori K, Sakai H, Nakai Y, Sugiura T, Kawahara $\mathrm{M}$ and Niitani $\mathrm{H}$ : S-1 plus cisplatin combination chemotherapy in patients with advanced non-small cell lung cancer: A multi-institutional phase II trial. Clin Cancer Res 10: 7860-7864, 2004

9. Kawahara M, Furuse K, Segawa Y, Yoshimori K, Matsui K, Kudoh S, Hasegawa K and Niitani H; S-1 Cooperative Study Group (Lung Cancer Working Group): Phase II study of S-1, a novel oral fluorouracil, in advanced non-small-cell lung cancer. Br J Cancer 85: 939-943, 2001.

10. Furuse K, Kawahara M, Hasegawa K, Kudoh S, Takada M, Sugiura T, Ichinose Y, Fukuoka M, Ohashi Y and Niitani H; S-1 Cooperative Study Group (Lung Cancer Working Group): Early phase II study of S-1, a new oral fluoropyrimidine, for advanced non-small-cell lung cancer. Int J Clin Oncol 6: 236-241, 2001.

11. Okamoto I, Yoshioka H, Morita S, Ando M, Takeda K, Seto T, Yamamoto N, Saka H, Asami K, Hirashima T, et al: Phase III trial comparing oral S-1 plus carboplatin with paclitaxel plus carboplatin in chemotherapy-naïve patients with advanced non-small-cell lung cancer: Results of a west Japan oncology group study. J Clin Oncol 28: 5240-5246, 2010.

12. Kubota K, Sakai H, Katakami N, Nishio M, Inoue A, Okamoto H, Isobe $\mathrm{H}$, Kunitoh $\mathrm{H}$, Takiguchi $\mathrm{Y}$, Kobayashi $\mathrm{K}$, et al: A randomized phase III trial of oral S-1 plus cisplatin versus docetaxel plus cisplatin in Japanese patients with advanced non-small-cell lung cancer: TCOG0701 CATS trial. Ann Oncol 26: 1401-1408, 2015.
13. Guo $\mathrm{Zw}$ and Gallo JM: Selective Protection of 2',2'-Difluorodeoxycytidine (Gemcitabine). J Org Chem 64: 8319-8322, 1999.

14. Ichinose Y, Seto T, Semba H, Itoh K, Inoue Y, Tanaka F, Araki J, Tamanoi M, Yamamoto H and Iwamoto N: UFT plus gemcitabine combination chemotherapy in patients with advanced non-small-cell lung cancer: A multi-institutional phase II trial. Br J Cancer 93: 770-773, 2005.

15. Nakamura K, Yamaguchi T, Ishihara T, Sudo K, Kato H and Saisho H: Phase II trial of oral S-1 combined with gemcitabine in metastatic pancreatic cancer. Br J Cancer 94: 1575-1579, 2006.

16. Kaira K, Sunaga N, Yanagitani N, Aoki H, Kawata T, Utsugi M, Shimizu Y, Shimizu K, Hisada T, Ishizuka T and Mori M: Phase I trial of oral S-1 plus gemcitabine in elderly patients with non-small cell lung cancer. Anticancer Drugs 19: 289-294, 2008.

17. Seto T, Yamanaka T, Wasada I, Seki N, Okamoto H, Ogura T, Shibuya M, Takiguchi Y, Shinkai T, Masuda N, et al: Phase I/II trial of gemcitabine plus oral TS-1 in elderly patients with advanced non-small cell lung cancer: Thoracic oncology research group study 0502. Lung Cancer 69: 213-217, 2010.

18. Satouchi M, Kotani Y, Katakami N, Shimada T, Urata Y, Yoshimura S, Funada Y, Hata A, Ando M and Negoro S: Randomized phase II study of two different schedules of gemcitabine and oral S-1 in chemo-naïve patients with advanced non-small cell lung cancer. J Thorac Oncol 5: 696-701, 2010.

19. Takiguchi Y, Seto T, Ichinose Y, Nogami N, Shinkai T, Okamoto H, Minato K, Seki N, Eguchi K, Kishi K, et al: Long-term administration of second-line chemotherapy with S-1 and gemcitabine for platinum-resistant non-small cell lung cancer. A phase II study. J Thorac Oncol 6: 156-160, 2011.

20. Therasse P, Arbuck SG, Eisenhauer EA, Wanders J, Kaplan RS, Rubinstein L, Verweij J, Van Glabbeke M, van Oosterom AT, Christian MC and Gwyther SG: New guidelines to evaluate the response to treatment in solid tumors. J Natl Cancer Inst 92: 205-216, 2000

21. Trotti A, Colevas AD, Setser A, Rusch V, Jaques D, Budach V, Langer C, Murphy B, Cumberlin R, Coleman CN and Rubin P: CTCAE v3.0: Development of a comprehensive grading system for the adverse effects of cancer treatment. Semin Radiat Oncol 13: 176-181, 2003.

22. Kudoh S, Takeda K, Nakagawa K, Takada M, Katakami N, Matsui K, Shinkai T, Sawa T, Goto I, Semba H, et al: Phase III study of docetaxel compared with vinorelbine in elderly patients with advanced non-small-cell lung cancer: Results of the West Japan Thoracic Oncology Group Trial (WJTOG 9904). J Clin Oncol 24: 3657-3663, 2006.

23. Gajra A and Jatoi A: Non-small-cell lung cancer in elderly patients: A discussion of treatment options. J Clin Oncol 32: 2562-2569, 2014.

24. Maione P, Perrone F, Gallo C, Manzione L, Piantedosi F, Barbera S, Cigolari S, Rosetti F, Piazza E, Robbiati SF, et al: Pretreatment quality of life and functional status assessment significantly predict survival of elderly patients with advanced non-small-cell lung cancer receiving chemotherapy: A prognostic analysis of the multicenter Italian lung cancer in the elderly study. J Clin Oncol 23: 6865-6872, 2005. 